\title{
An Application of Non-Uniform Cellular Automata for Efficient Cryptography
}

\author{
A. Kumaravel ${ }^{1 *}$ and Oinam Nickson Meetei ${ }^{2}$ \\ 1 Dean \& Professor, Department of Computer Science \& Engineering \\ Bharath University, Chennai; drkumaravel@gmail.com \\ ${ }^{2}$ PG Scholar, Department of Computer Science \& Engineering \\ Bharath University, Chennai; nickson_s@hotmail.com
}

\begin{abstract}
One of the core issues for robust cryptographic systems is to deal with the rate of diffusion and distribution of keys. We try to strengthen this aspect by increasing the length of block ciphers. We present a new encryption and decryption algorithm for block cipher that supports $2^{7}$ bit block size. All components in our system are chosen to be based on cellular automata so as to achieve higher parallelism and to simplify the in hardware and software implementation for applications with high degree of security. The main objective of this paper is to increase the complexity by novel schemes of mixing (reversible cellular automata) RCA and (non-uniform reversible cellular automata) NRCA. We apply set of different bit permutation methods for this purpose. This paper establishes the proof for existence of yet another approach for a high quality pseudorandom bit sequences generated by non uniform cellular automata.
\end{abstract}

Keywords: Cryptographic System, NRCA-non Reversible CA, Pseudorandom Number, RCA-Reversible Cellular Automata.

\section{Introduction}

Two main cryptography systems are used today: symmetric systems (aka secret key systems) and public-key systems. An extensive overview of currently known or emerging cryptography techniques used in both type of systems can be found in $[1,9]$. One of such a promising cryptography technique is applying cellular automata (CAs).

The main concern of this paper is secret key systems. In such systems the encryption key and the decryption key are same (symmetric key). The encryption process is based on generation of pseudorandom bit sequences, and CAs can be effectively used for this purpose. Cellular Automata (CA) is an organized lattice of cells and each cell have finite number of states, such as "TRUE" (T) or "FALSE" (F). The lattice dimensions can be of any finite value. Each cell within a collection of cells is called as hood. It is characterized

\footnotetext{
* Corresponding author:
}

A. Kumaravel (drkumaravel@gmail.com) relatively with respect to a particular cell. To start with at time $t=0$, a state is assigned to the cells. The new states of the cell depend on its own previous state and states of its neighborhood. The new states are assigned based on some predefined rule using mathematical calculations.

Encryption, by theory requires highly complex actions such as permuting, flipping and altering data in such a way that it is undecipherable and provides complex relationship with the original text and the keys. This relationship should be non-linear so that decryption is as tough as possible. The encryption process must be faster in time and cheaper in terms of the components involved [2,7]. CA provides a basic structure for highly parallel and complex operations upon which a basic encryption scheme can be built. The message encryption is done by Pseudo Random Number Generators (PRNGs) using CA. The generation of new states in OneDimensional (1-D) CA, can be considered as a sequence of 
random numbers [3]. Different security schemes have been proposed including symmetric key, hash functions and public key cryptography as observed by Sarkar, [3]. Further as stated by Wolfram $[4,5]$, Rule 30 promotes the use of large integers in the pseudo random number generation. Owing to this interesting chaotic property of the peculiar CA, Wolfram states that, this kind of CA is used as random number generator.

The structure of the paper is as follows: Section II describes studies of symmetric key algorithms and its performances; Section III depicts our proposed algorithm in detail. Finally, conclusion is expressed.

\section{Terms, Models and Methods}

Here we discuss the terminology used in the paper for easier reading.

\subsection{Lightweight Cellular Automata Symmetric-key Encryption}

One-dimensional cellular automata encryption algorithm called as Lightweight Cellular Automata-based Symmetrickey Encryption (LCASE) is described in [2]. Here rule 30 is used for encrypting plain-text with keys. The fundamental design objective of LCASE is to effectively optimize the performance requirements of both software and hardware. However, the algorithm also has to address the conventional security constraints. Different issues considered for designing the proposed algorithm are:

a. High speed performance and minimum code density

b. Defiant to attack such as conventional cryptanalysis and timing attack

c. Simple and code effective implementation

The implementation of cipher in either of hardware or software has to satisfy two important design considerations [2]. The algorithm should be fast and easy to implement. In order to address these constraints parallel processing methods are used. CA elements are found to be effective in the design process. Number of loops used in LCASE consists of two rounds namely, 1. Last-half (LH) 2. First- half (FH). The term ' $r$ ' refers to a complete round and ' $r+1$ ' round refers to $\mathrm{FH}$. The selection of ' $r$ ' value is shown in the Table 1.

This encryption is a reversible two way process, decryption is obtain as the result of this. Each encryption round uses 1-D (3-neighbourhood) 32 bits periodic boundary CA. The
Table 1. Selection of ' $r$ ' value

\begin{tabular}{llll}
\hline Keys size in bits & 128 & 192 & 156 \\
'r' value & 12 & 14 & 16 \\
\hline
\end{tabular}

Table 2. Selection of rule for different operations

\begin{tabular}{ll}
\hline Operation & Rule \\
\hline RCA(second order) & 30 \\
& Skewed 30 \\
& Skewed 45 \\
NCA & 218 \\
\hline
\end{tabular}

basic element of a round uses Reversible Cellular Automata (RCA), Bit Permutation (BP), Non-autonomous Cellular Automata (NCA) and Reverse Substitution (RS). The rules used in LCASE for different operations are given in Table 2.

In order to effectively obtain the non linearity property, suitable rules from Table 2 have to be chosen for the selected operators. In this cipher, RCA and NCA are the preferred operators over the conventional XOR, as the later is not suitable for non linear operations.

\section{Main Algorithm}

We construct the main algorithm based on following terminologies.

\subsection{Next State Generation by Various Ca Rules}

Our proposed method uses CA rules 30, 45,218 as mentioned as in [2] and 86, 90,105,150,165 as in [6] for NRCA and RCA operation. The next state generation of these 8 rules is given in Table 3 .

Table 3. Next State Configuration for CA Rules 30, 45, $218,90,150,145,165$ and 86

Neigh- $\quad \begin{array}{llllllllll}111 & 110 & 101 & 100 & 011 & 010 & 001 & 000 & \text { Rule No. }\end{array}$ borhood

State:

\begin{tabular}{|c|c|c|c|c|c|c|c|c|}
\hline Next State : 0 & 0 & 0 & 1 & 1 & 1 & 1 & 0 & 30 \\
\hline Next State: 0 & 0 & 1 & 0 & 1 & 1 & 0 & 1 & 45 \\
\hline Next State: 0 & 1 & 0 & 1 & 0 & 1 & 1 & 0 & 86 \\
\hline Next State : 0 & 1 & 0 & 1 & 1 & 0 & 1 & 0 & 90 \\
\hline Next State: 0 & 1 & 1 & 0 & 1 & 0 & 0 & 1 & 105 \\
\hline Next State : 1 & 0 & 0 & 1 & 0 & 1 & 1 & 0 & 150 \\
\hline Next State: 1 & 0 & 1 & 0 & 0 & 1 & 0 & 1 & 165 \\
\hline Next State : 1 & 1 & 0 & 1 & 1 & 0 & 1 & 0 & 218 \\
\hline
\end{tabular}




\subsection{RCA Key Schedule}

RCA is the one in which the preceding pattern can be recovered from the given current pattern (s). Our proposed algorithm utilizes RCA (second order) [10]. The ' $\mathrm{i}^{\text {th' }}$ state of $\mathrm{n}$ bit pattern is determined by the clock cycles as shown in Table 4. Given below is an example of a 3-neighborhood second order RCA [2]

$$
\begin{aligned}
& x_{i}(t-1)=f\left(x_{i-1}(t),\left(x_{i}(t), x_{i+1}(t)\right)\right) X O R x_{i}(t+1) \\
& x_{i}(t+1)=f\left(x_{i-1}(t),\left(x_{i}(t), x_{i+1}(t)\right)\right) X O R x_{i}(t-1)
\end{aligned}
$$

Here, the states $\mathrm{x}_{\mathrm{i}}(\mathrm{t}+1)$ and $\mathrm{x}_{\mathrm{i}}(\mathrm{t}-1)$ are denoted respectively by the terms $\xi_{i}$ and $y_{i}$ and ' $f$ ' is from Table 5 .

$\xi$ is obtained based on two initial patterns of $(\mathrm{Y}, \mathrm{X})$ at time steps $(t-1)$ and $t$. Then, using two successive patterns $(\xi, X)$, the initial pattern Y can be figured out. This operation is denoted as follows [2].

$$
\xi=\operatorname{RCA}(\mathrm{Y}, \mathrm{X}) ; \mathrm{Y}=\operatorname{RCA}(\xi, \mathrm{X}) .
$$

Elementary CA rule 30 based second order periodic boundary 4-cell RCA logic diagram is depicted in Figure 1.

The RCA key schedule generates 16 bytes of key for each encryption and decryption round. For each round of key generation we applied different rule which is selected sequentially from Table 5. RCA key schedule is explained in "Figure 2" and the RCA operation between two 8 bits

\begin{tabular}{|c|c|}
\hline Clock cycles & ' $i^{\text {th' }}$ state of $n$ bit pattern \\
\hline$t+1$ & $\begin{array}{l}\text { States of neighbourhood pattern at ' } \mathrm{t} \text { ' \& current } \\
\text { cell at }(\mathrm{t}-1)\end{array}$ \\
\hline$t-1$ & patterns at $\mathrm{t} \&(\mathrm{t}+1)$ clock cycle \\
\hline
\end{tabular}
sub- block is shown in Figure 3.

Table 4. State Determination

\begin{tabular}{|c|c|}
\hline Rule No. & Next state function(f) \\
\hline 30 & $x_{i}(t+1)=x_{i}-1(t) X O R\left(x_{i}(t) \vee x_{i+1}(t)\right)$ \\
\hline 45 & $\left.\mathrm{x}_{\mathrm{i}}(\mathrm{t}+1)=\mathrm{x}_{\mathrm{i}-1}(\mathrm{t}) \operatorname{XOR}\left(\mathrm{x}_{\mathrm{i}}(\mathrm{t}) \vee \mathrm{x}\right\urcorner_{\mathrm{i}+1}(\mathrm{t})\right)$ \\
\hline 86 & $\mathrm{x}_{\mathrm{i}}(\mathrm{t}+1)=\mathrm{x}_{\mathrm{i}} \neg(\mathrm{t}) \wedge\left(\mathrm{x}_{\mathrm{i}-1}(\mathrm{t}) \mathrm{XOR} \mathrm{x}_{\mathrm{i}+1}(\mathrm{t})\right) \vee \mathrm{x}_{\mathrm{i}}(\mathrm{t}) \wedge \mathrm{x} \neg_{\mathrm{i}+1}(\mathrm{t})$ \\
\hline 90 & $\mathrm{x}_{\mathrm{i}}(\mathrm{t}+1)=\mathrm{x}_{\mathrm{i}-1}(\mathrm{t}) \operatorname{XOR} \mathrm{x}+1_{\mathrm{i}+1}(\mathrm{t})$ \\
\hline 105 & $x_{i}(t+1)=x_{i}(t) \operatorname{XNOR}\left(x_{i-1}(t) X O R x_{i+1}(t)\right)$ \\
\hline 150 & $x_{i}(t+1)=x_{i-1}(t) X O R x_{i}(t) X O R x_{i+1}(t)$ \\
\hline 165 & $x_{i}(t+1)=x_{i-1}(t) X_{N O R} x_{i+1}(t)$ \\
\hline 218 & $\mathrm{x}_{\mathrm{i}}(\mathrm{t}+1)=\left(\mathrm{x}_{\mathrm{i}}(\mathrm{t}) \wedge\left(\mathrm{x}_{\mathrm{i}+1}(\mathrm{t})\right) \vee\left(\mathrm{x}_{\mathrm{i}-1}(\mathrm{t}) \mathrm{XOR}_{\mathrm{i}+1}(\mathrm{t})\right)\right.$ \\
\hline
\end{tabular}

Table 5. Next State Function for cellular Automata Rules from Table 3

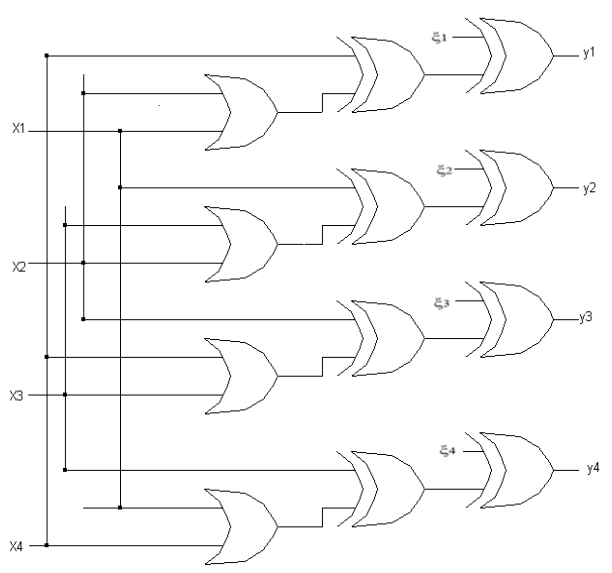

Figure 1. Combintiraal circuit for 4-cell periodic boundary RCA based on. Rule 30, represented by the state equation $\mathrm{x}_{\mathrm{i}}(\mathrm{t}+1)=\mathrm{f}\left(\mathrm{x}_{\mathrm{i}-1}(\mathrm{t}) \operatorname{XOR}\left(\mathrm{x}_{\mathrm{i}}(\mathrm{t}) \vee \mathrm{x}_{\mathrm{i}+1}(\mathrm{t})\right)\right) \operatorname{XOR} \mathrm{x}_{\mathrm{i}}(\mathrm{t}-1)$

\section{Pseudocode:}

Pseudocode for RCA key schedule in Figure 2.

Input: $\mathrm{K}\left(\mathrm{k}_{\mathrm{i}}: \mathrm{i}:=1\right.$ to 16$) ; / / \mathrm{K}=$ user selection 128 bits key and $\mathrm{k}_{1}, \mathrm{k}_{2} \ldots \ldots, \mathrm{k}_{16}$ are 8 bits words//

Output: $\mathrm{k}_{\mathrm{i}}^{\mathrm{L}}(\mathrm{i}=1$ to 16$) ; / / \mathrm{k}_{1}^{\mathrm{L}}, \mathrm{k}_{2}{ }_{2}^{\mathrm{L}}, \ldots \ldots, \mathrm{k}_{16}{ }^{\mathrm{L}}$ are 8 bits words key schedule generated for $\mathrm{L}^{\text {th }}$ round//

$\mathrm{L}=$ number of rounds

BEGIN

$$
\begin{aligned}
& \text { // computation of encryption key// } \\
& \text { for } \mathrm{r}:=1 \text { to } \mathrm{L}-1 \text { do } \\
& \text { for } \mathrm{i}:=1 \text { to } 16 \text { do } \\
& \text { if }(\mathrm{i}:=16) \text { do } \\
& \quad \mathrm{K}_{\mathrm{i}}^{\mathrm{r}+1}:=\mathrm{RCA}\left(\mathrm{K}_{\mathrm{i}}^{\mathrm{r}}, \mathrm{K}_{1}^{\mathrm{r}}\right) \text {; } \\
& \text { endif } \\
& \text { if }(\mathrm{i}>16) \text { do } \\
& \mathrm{K}_{\mathrm{i}}^{\mathrm{r}+1}:=\mathrm{RCA}\left(\mathrm{K}_{\mathrm{i}}^{\mathrm{r}}, \mathrm{K}_{1+1}{ }^{\mathrm{r}}\right) \text {; } \\
& \text { endif } \\
& \text { endfor } \\
& \text { endfor }
\end{aligned}
$$

//Determination of decryption key //

$$
\begin{aligned}
& / / \mathrm{DK}=\text { decryption key and } \mathrm{k}=\text { encryption key// } \\
& \text { For } \mathrm{r}:=1 \text { to } 16 \text { do } \\
& \quad \mathrm{DK}_{1}^{\mathrm{r}}:=\mathrm{k}_{1}^{|\mathrm{r}-(\mathrm{L}+1)| ;} \\
& \text { endfor } \\
& \text { END }
\end{aligned}
$$

//For each round of encryption and decryption the RCA key schedule generates sixteen keys each of eight bits.

The next key schedule is generated from the previous key schedule.// 


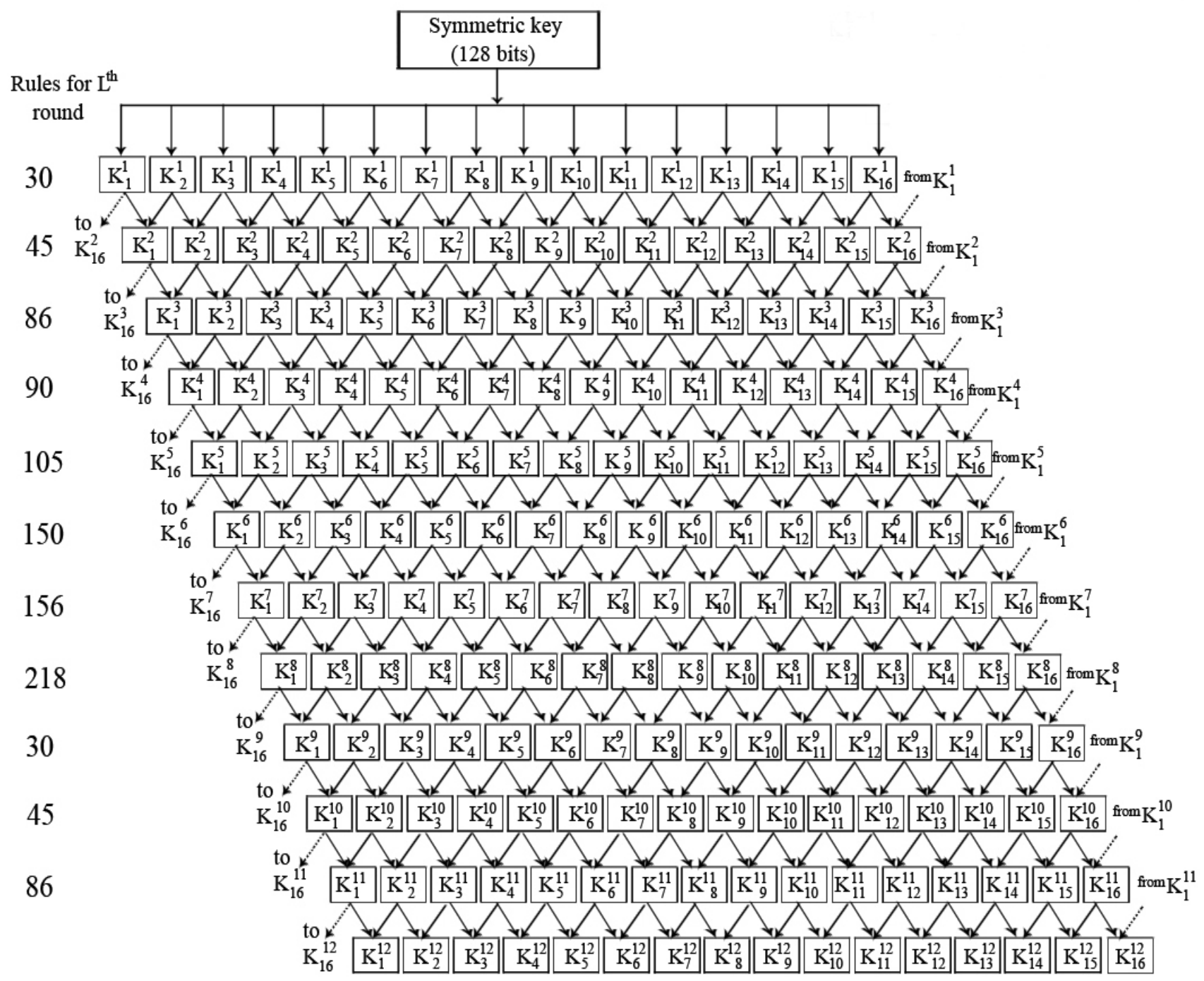

$\mathrm{K}_{\mathrm{i}}^{\mathrm{L}}=\mathrm{i}^{\text {th }} 8$ bits sub block for $\mathrm{L}^{\text {th }}$ round.

Figure 2. RCA key schedule.

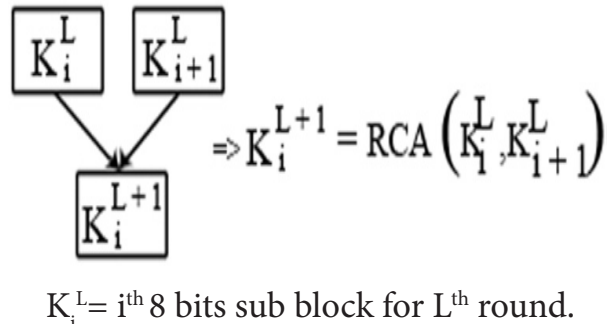

Figure 3. RCA operation between two 8 bits sub- block.

\subsection{Modulo Prime Operation for}

\section{Permutation}

The Bit-Permutation (BP (input[32 bits])) operation permutes the $i^{\text {th }}$ bit into $\left(\left(9^{*} \mathrm{i} \bmod 31\right)+1\right)^{\text {th }}$ bit. The idea behind this permutation is to place the three consecutive bits into three different locations. This increases the rate of diffusion and makes differential cryptanalysis very complex for our encryption scheme. Moreover the realization of permutation is obtained by connecting wire crossings appropriately.

Figure 4 shows an example of bit permutation which involves 4 bytes (32 bits). Here the three neighborhoods bits in position 1,2 and 3 are transferred into 10,19 and 28 positions.

\subsection{Non-Uniform Reversible Cellular Automata}

NRCA is a RCA operation in which we applied 8 different rules to each bit of each byte from Table 5. Each bit 
being applied with a unique rule. The transition function are computed parallel in all bits as shown in the Figure 5 Each block receives 8 bits and produces 8 bits.

\subsection{Reversible Bit Permutation (RBP)}

The Reversible Bit-Permutation (RBP) operation is the process of re-permuting the distributed bits into its original position. The idea behind is to restore original position.

Figure 4 shows an example of bit permutation which involves 4 bytes, in RBP, the three distributed bit in position 10,19 and 28 is transfer into its original position that is 1, 2 and 3 position as shown in Figure 6.

Algorithm for Reversible bit permutation.

Step 1: Assign $\mathrm{j}$ from 1 to 31 in the input word RBP input [32 bits]

Step 2: Compute $k$ as $\left(\left(9^{*} \mathbf{j} \bmod 31\right)+1\right)$

Step 3: RBP output $[\mathrm{j}]=\mathrm{RBP}$ input $[\mathrm{k}]$

\section{Input Bit Position}
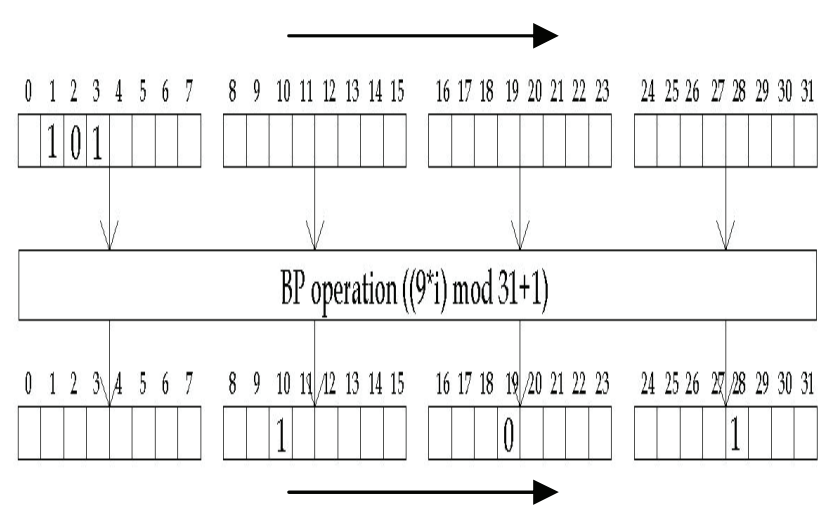

Output Bit Position

Figure 4. Bit permutation in 32 bits.

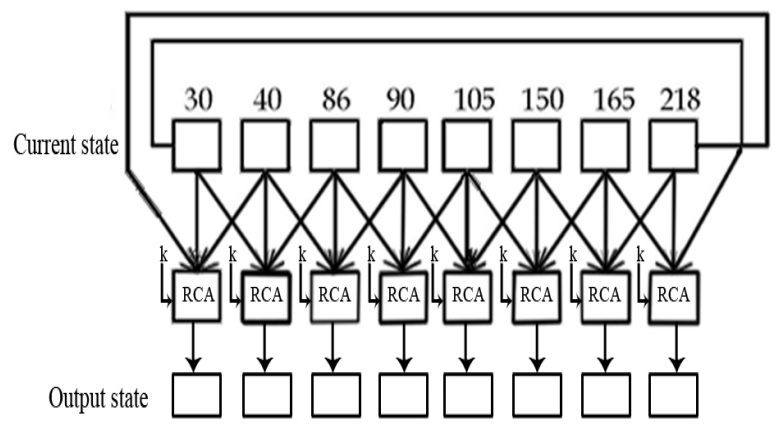

$30,40,86,90,105,150,165$ are rules use in RCA

$\mathrm{K}=$ encryption key for the round for the block

Figure 5. NRCA operation in 8 bits block.
Output Bit Position

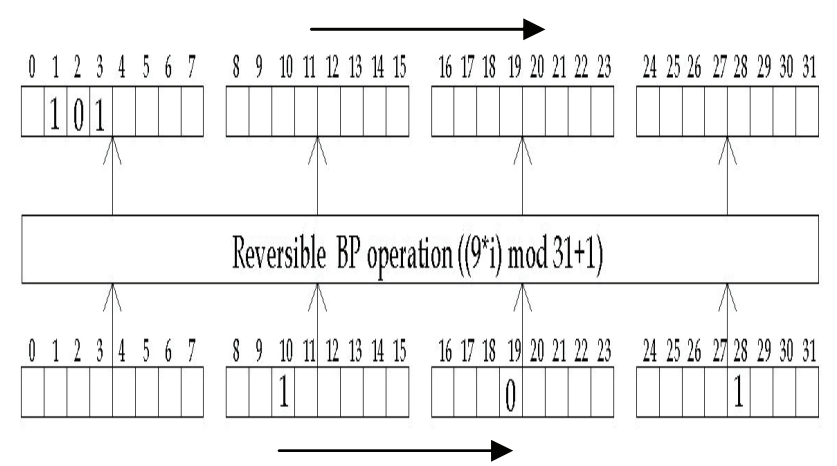

Input Bit Position

Figure 6. Reversible bit permutation.

\subsection{Main algorithm}

Using the above method we describe the encryption process with one-dimensional reversible cellular automata (1D RCA) as shown in Figure 7. Our entire encryption round comprises simple XOR, Bit Permutation, RCA key schedule, NRCA. The ' $r$ ' values are chosen from the Table 1. Here we used 8 different rules from Table 3 for NRCA and RCA operation. Each encryption round uses the 8 different rules to each byte for NRCA operation. In RCA key generator, for each encryption round a rule is selected sequentially in cyclic order from the Table 3 for generating next key.

\subsection{Decryption}

Since we used RCA (reversible cellular automata), NRCA(non-uniform RCA), XOR and reversible BP(bit permutation), we can decrypt the cipher text to plain text in reverse way using the decryption key which is explained in pseudo code of RCA key schedule. The cipher text can be decrypted from the cipher text and the key. The computational flow of decryption scheme is given in Figure 8.

\section{Conclusion}

The above routines are tested and we have comparable results based on these proposed algorithms. In this paper we have tried with a light weight cryptographic algorithm based on symmetric key based on non-uniform cellular automata and dynamic key generation. This approach decreases the space requirements especially for small devices and increases the better results with the rate of 


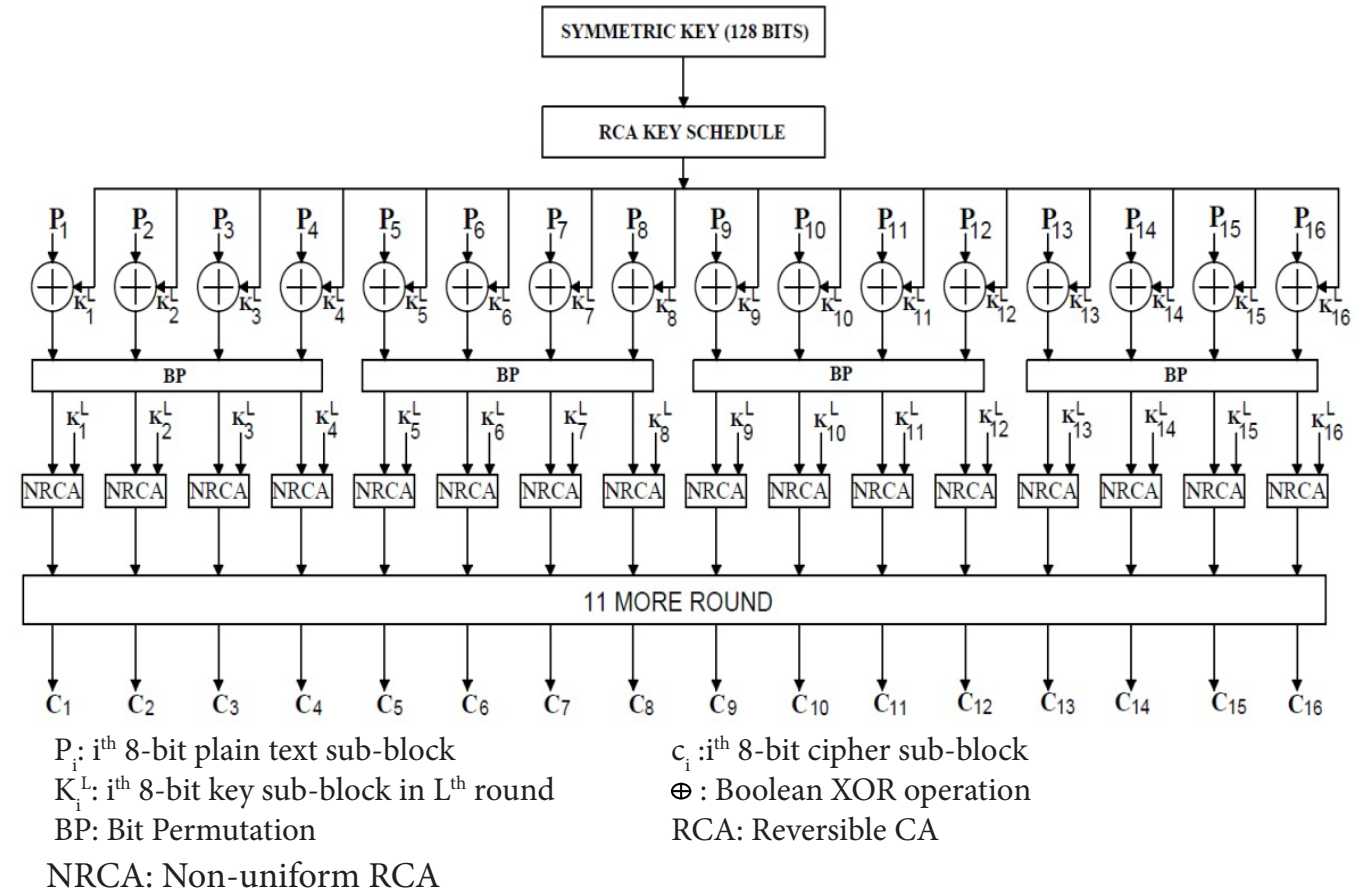

Figure 7. 128 key bits Encryption scheme applying CA rules.

Pseudocode:

Pseudo code for realising the CA rules found in Figure 6.

// Uppercase alphabet set represent 128 bit block and corresponding lowercase sets specify 8 bit words.//

Input:Plaintext block, $\mathrm{P}\left(\mathrm{p}_{\mathrm{i}}: \mathrm{i}=1\right.$ to 16$)$;

$$
\text { K: User } 128 \text { bits key; }
$$

Output: Cipher block, $\mathrm{C}\left(\mathrm{c}_{\mathrm{i}:} \mathrm{i}=1\right.$ to 16$)$;

Intermediate cipher text : $\mathrm{IC}\left(\mathrm{Ic}_{\mathrm{i}} \mathrm{i}=1\right.$ to 16$)$;

$\mathrm{BP}_{1}, \mathrm{BP}_{2}, \mathrm{BP}_{3}, \mathrm{BP}_{4}$ are 32 bits words

$\mathrm{bp}_{1}, \mathrm{bp}_{2} \ldots \ldots \ldots \mathrm{bp}_{16} 8$ bits words

\section{BEGIN}

READ $p_{i} ;$

READ K;

//Apply RCA key schedule//

IC:=P;

Keyschedule $\left(\mathrm{k}_{\mathrm{i}}\right)$, (i=1 to 16$)$; //16 8 bits block gen-

erated by key generator for $\mathrm{L}^{\text {th }}$ round//

For $\mathrm{L}:=1$ to end of round do

$\mathrm{S}_{1}:=\mathrm{Ic}_{1} \oplus \mathrm{k}_{1} ; \mathrm{S}_{2}:=\mathrm{Ic}_{2} \oplus \mathrm{k}_{2} ; \mathrm{S}_{3}:=\mathrm{Ic}_{3} \oplus \mathrm{k}_{3} ;$

$\mathrm{S}_{4}:=\mathrm{Ic}_{4} \oplus \mathrm{k}_{4} ; \mathrm{S}_{5}:=\mathrm{Ic}_{5} \oplus \mathrm{k}_{5} ; \mathrm{S}_{6}:=\mathrm{Ic}_{6} \oplus \mathrm{k}_{6} ;$

$\mathrm{S}_{7}:=\mathrm{Ic}_{7} \oplus \mathrm{k}_{7} ; \mathrm{S}_{8}:=\mathrm{Ic}_{8} \oplus \mathrm{k}_{8} ; \mathrm{S}_{9}:=\mathrm{Ic}_{9} \oplus \mathrm{k}_{9} ;$

$\mathrm{S}_{10}:=\mathrm{Ic}_{10} \oplus \mathrm{k}_{10} ; \mathrm{S}_{11}:=\mathrm{Ic}_{11} \oplus \mathrm{k}_{11} ; \mathrm{S}_{12}:=\mathrm{Ic}_{12} \oplus \mathrm{k}_{12} ;$

$\mathrm{S}_{13}:=\mathrm{Ic}_{13} \oplus \mathrm{k}_{13} ; \mathrm{S}_{14}:=\mathrm{Ic}_{14} \oplus \mathrm{k}_{14} ; \mathrm{S}_{15}:=\mathrm{Ic}_{15} \oplus \mathrm{k}_{15}$;

$$
\mathrm{S}_{16}:=\mathrm{Ic}_{16} \oplus \mathrm{k}_{16}
$$

// create four $\mathrm{BP}_{\mathrm{i}} / /$

$$
\begin{aligned}
& \mathrm{BP}_{1}:=\mathrm{BP}\left(\mathrm{S}_{1}, \mathrm{~S}_{2}, \mathrm{~S}_{3}, \mathrm{~S}_{4}\right) ; / / \mathrm{S}_{1, \ldots . . .} \mathrm{S}_{16} \text { are } 8 \text { bits wards// } \\
& \mathrm{BP}_{2}:=\mathrm{BP}\left(\mathrm{S}_{5}, \mathrm{~S}_{6}, \mathrm{~S}_{7}, \mathrm{~S}_{8}\right) \text {; } \\
& \mathrm{BP}_{3}:=\mathrm{BP}\left(\mathrm{S}_{9}, \mathrm{~S}_{10}, \mathrm{~S}_{11}, \mathrm{~S}_{12}\right) \text {; } \\
& \mathrm{BP}_{4}:=\mathrm{BP}\left(\mathrm{S}_{13}, \mathrm{~S}_{14}, \mathrm{~S}_{15}, \mathrm{~S}_{16}\right) \text {; } \\
& \text { Ic }:=\operatorname{NRCA}\left(\mathrm{bp}_{\mathrm{i}}, \mathrm{k}_{\mathrm{i}}\right) \text {; } \\
& \text { end loop } \\
& c_{i}:=I c_{i} \text {; }
\end{aligned}
$$




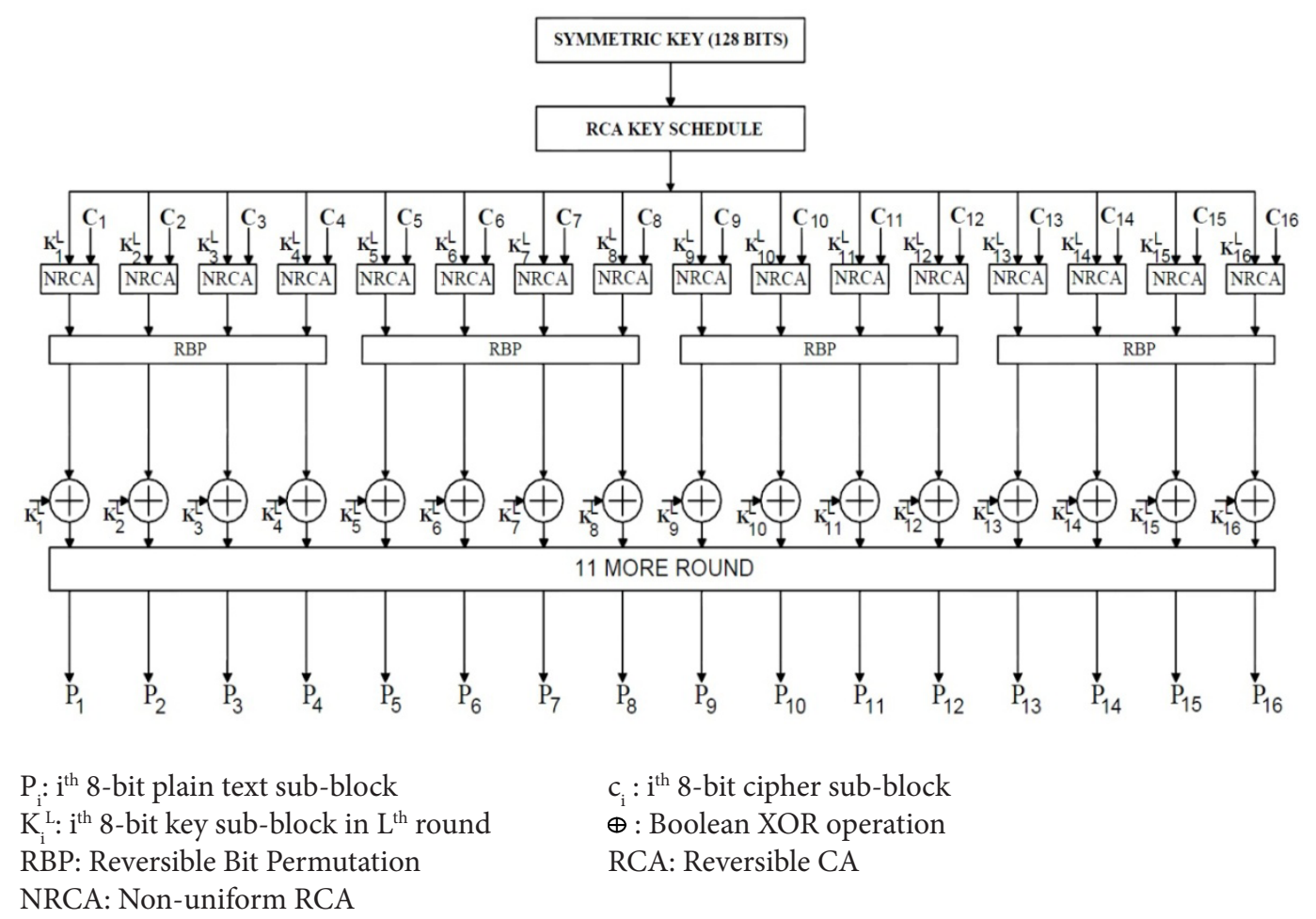

Figure 8. 128 key bits Decryption scheme applying CA rules.

diffusion and distribution of keys. Moreover the different rule of neighborhood has been applied for cellular at each byte position for encryption and decryption. Hence our proposed method strengthens the simplicity for establishing the implementation in software and hardware.

\section{References}

1. Schneier B (1996). Applied cryptography, Wiley, New York.

2. Tripathy S, and Nandi S (2009). LCASE: Lightweight Cellular Lightweight Cellular Automata-based Symmetrickey Encryption, International Journal of Network Security, vol 8(2), 243-252.

3. Sarkar P (2000). A brief history of cellular automata, Journal of ACM Computing Surveys (CSUR), vol 32(1), 80-107.

4. Wolfram S (1986). Cryptography with cellular automata, Crypto '85, LNCS 218, Springer-verlag, 429-432.
5. Wolfram S (1986). Random sequence generation by cellular automata, Advances in Applied Mathematics, vol 7(2), 123169.

6. Seredynski F, Bouvry P et al. (2004). Cellular automata computations and secret key cryptography, Parallel Computing Journal, vol 30(5-6), 753-766.

7. Standaert F, Piret G et al. (2004). ICEBERG : An involutional cipher efficient for block encryption in reconfigurable hard- ware, FSE '04, LNCS 3017, Springer- verlag, 279-299.

8. Sklavos N, Moldovyan N A et al. (2005). High speed networking security: Design and implementation of two new DDP-based ciphers, Mobile Networks and ApplicationsMONET, vol 25(1-2), 219-231.

9. Moldovyan N A, Moldovyan P A et al. (2007). On software implementation of fast DDP-based ciphers, International Journal of Network Security, vol 4(1), 81-89.

10. Toffoli T, and Margolus N (2001). Invertible cellular automata: A review, Physica D, vol 45(1-3), 229-253, (Reprinted with correction as of $\mathrm{Oct}$ ). 